Vol. 45 (1992) [351-351]

\title{
On mathematical modelling of the self-heating of cellulosic materials ROBERT ANTHONY SisSON
}

This thesis considers mathematical modelling of self-heating of cellulosic materials, and in particular the effects of moisture on the heating characteristics. Following an introductory chapter containing a literature review, Chapter 2 presents some preliminary results and an industrial case study. The case study, which discusses a 'dry' body self-heating on a hot surface, investigates the following questions:

(i) how hot can the surface get before ignition is likely?

(ii) how well does the (slab-like) body approximate to an infinite slab?

(iii) how valid is the Frank-Kamenetskii approximation for the source term?

It is shown that the minimal steady state temperature profile is stable when the temperature of the hot surface is below a certain critical value, and bounds for the higher steady state profile are derived. Chapter 3 presents the thermodynamic derivation of a reaction-diffusion model for the self-heating of a moist cellulosic body, including the effects of direct chemical oxidation as well as those of a further exothermic hydrolysis reaction and the evaporation and condensation of water. The model contains three main variables: the temperature of the body, the liquid water concentration in the body, and the water vapour concentration in the body. Chapter 4 investigates the limiting case of the model equations as the thermal conductivity and diffusivity of the body become large. In particular it is shown that, in this limiting case, the model can have at least twenty-five distinct bifurcation diagrams, compared with only two for the well known model without the effects of moisture content. In Chapter 5 the maximum principle and the methods of upper and lower solutions are used to derive existence, uniqueness and multiplicity results for the steady state solutions of the spatially distributed model. Finally, in Chapter 6, existence and uniqueness results for the time dependent spatially distributed model are derived.

\author{
Department of Mathematics \\ Massey University \\ Palmerston North \\ New Zealand
}

Received 23rd September 1991

Thesis submitted to Massey University, June 1991. Degree approved August 1991. Supervisors: Professor Graeme Wake and Mr Adrian Swift.

Copyright Clearance Centre, Inc. Serial-fee code: 0004-9729/92 \$A2.00+0.00. 